\title{
Soziale Ungleichheiten als Ursprung technologischer Diskrepanzen
}

Partha Pratim Sarker

\section{(2) OpenEdition}

\section{Journals}

Electronic version

URL: http://journals.openedition.org/sjep/524

DOI: $10.4000 /$ sjep.524

ISSN: 1663-9677

\section{Publisher}

Institut de hautes études internationales et du développement

\section{Printed version}

Date of publication: 1 novembre 2003

Number of pages: $37-39$

ISSN: 1660-5926

\section{Electronic reference}

Partha Pratim Sarker, «Soziale Ungleichheiten als Ursprung technologischer Diskrepanzen », Schweizerisches Jahrbuch für Entwicklungspolitik [Online], 22-2 | 2003, Online erschienen am: 10 Juni 2010, abgerufen am 08 September 2020. URL : http://journals.openedition.org/sjep/524 ; DOI : https://doi.org/10.4000/sjep.524 


\title{
Soziale Ungleichheiten als Ursprung technologischer Diskrepanzen
}

\author{
Partha Pratim Sarker*
}

$\mathrm{B}$ eim digitalen Graben geht es nicht nur um Anschlussraten, sondern auch um Inhalt, Besitz, Beteiligung, soziale Einbeziehung usw.; eine ganze gesellschaftliche Machtstruktur ist ebenfalls mit dem digitalen Graben verbunden. Wenn wir diesen Graben überwinden wollen, müssen wir uns zuerst einmal vorrangig mit den Wurzeln der Ausbeutung und der Ressourcenverteilung in den betreffenden Gesellschaften befassen. Jedoch nehmen in den Entwicklungsländern die zur Verringerung des digitalen Grabens getroffenen Massnahmen eine ganz andere Ausrichtung an. Man befasst sich nur mit dem Zugang - indem man lediglich die Anzahl von Computern, Netzwerken und Systemen erhöht - ohne den gesellschaftlichen Prozess des Grabens umzuwandeln oder aufzuhalten. Interessanterweise fördern die entwickelten Länder und die Geberorganisationen diese Politiken, da sie in ihre globalen Handelsinteressen hineinpassen. Shahidul Alam stimmt hiermit überein, wenn er feststellt: „Da wo Information Macht ist, hindert die Verweigerung von Information für marginalisierte Gemeinschaften die Armen in den ländlichen Gebieten daran, die ungleichen Machtstrukturen $\mathrm{zu}$ überwinden, in denen sie gefangen sind. Während es im Interesse der Mächtigen in der Gesellschaft ist, einen solchen Zugang zu beschränken, ist es auch im Interesse der mächtigen Nationen, den Zugang zu verweigern und ihre Herrschaft aufrechtzuerhalten"1.

Einer der Schlüsselbereiche der Armutsbekämpfung, bei dem die Informations- und Kommunikationstechnologien (IKT) eine wichtige Rolle spielen können, hängt mit der Fähigkeit dieser Technologien zusammen, den Armen Gehör zu verschaffen und ihnen mehr Informationen und wirtschaftliche Möglichkeiten für ein besseres Leben zu bieten. In diesem Sinne kann ein Mangel an Zugang zu den IKT ein Hindernis für die wirtschaftliche, soziale und kulturelle Entwicklung sein, da dieser „Mangel“ den Zutritt zu wirtschaftlichen und politischen Möglichkeiten hinauszögert und beschränkt. Dies bedeutet jedoch nicht, dass ein angemessener Zugang zur Informationstechnologie automatisch zu wirtschaftlicher, sozialer und kultureller Entwicklung führen wird, sondern dass er unbestreitbar eine Chance für arme und abseits gelegene Gemeinschaften darstellt, die sich andernfalls in einer zeitaufwendigen, kostspieli- 
gen und komplizierten Art und Weise mit dem Markt oder der Gesellschaft $\mathrm{zu}$ verbinden hätten.

Die Umsetzung technologischer Aufholpolitik allein kann nicht die gewünschte wirtschaftliche Entwicklung für ein Land mit sich bringen, sofern nicht gleichzeitig noch einige andere Entwicklungen erfolgen. Die IKT können als Werkzeug zur Ausrottung der Armut eingesetzt werden, aber nicht als Alternative oder Ersatz für andere entwicklungsfördernde Massnahmen dienen. Stattdessen können die IKT oder die technologische Aufholpolitik solche Massnahmen verstärken oder erleichtern, wenn sie richtig integriert sind. Hier gibt es keine ,entweder - oder“ Situation. So wird zum Beispiel die Auswirkung eines verbesserten IKT-Zugangs auf die Einnahmen der Landwirte durch eine bessere Kenntnis der Marktpreise nutzlos sein, wenn es keine Strassen gibt, um die Ernte auf den Markt zu bringen, oder wenn es mangels anderer Infrastruktureinrichtungen keine Märkte gibt. Wie von Richard Heeks erwähnt, benötigen die Armen eher Wissen für den Zugang zu bereits bestehenden Informationen und Ressourcen, - um aufgrund dieser Informationen Massnahmen zu ergreifen - als dass sie Zugang zu neuen Informationen brauchen. Heeks vermerkt: „Informationsdefizite sind sicherlich ein Problem für arme Unternehmer, doch ein wichtigerer Teil des Gesamtbildes - und eine Voraussetzung für die Nutzung der Informationen - sind Ressourcen wie Fertigkeiten, Wissen und Geld“. ${ }^{2}$
Auf dem indischen Subkontinent habe ich viele Organisationen gesehen, welche zuvor in anderen Entwicklungsbereichen gearbeitet haben und nun zu Tätigkeitsgebieten übergegangen sind, die auf eine Überwindung des digitalen Grabens abzielen, nur weil so viele Finanzmittel zur Verfügung stehen. Nicht Engagement, nicht einmal Expertise oder Interesse bringen sie dazu, sich mit der Frage des digitalen Grabens zu befassen, sondern einfach die Verfügbarkeit finanzieller Mittel. Diese Tendenz nimmt jetzt in den Entwicklungsländern problematische Ausmasse an. Deshalb denke ich, dass es auch wichtig ist, die Nachhaltigkeit, Langlebigkeit und die massstäbliche Anpassbarkeit der Projekte zu untersuchen, bevor wir ein allgemeines Urteil abgeben und viel Aufhebens um sie machen. Zahlreiche Initiativen, die plötzlich auftauchen und ansehnliche Auszeichnungen erhalten, verschwinden im Lauf der Zeit wieder.

Die privaten Unternehmen werden sich auf kein Geschäft einlassen, wenn sie nicht dahinter einen wirtschaftlichen Nutzen oder einen Gewinn sehen. Ich stimme persönlich nicht mit dem Argument überein, dass es an ländlichen oder entlegenen Orten (wo der digitale Graben am stärksten ausgeprägt ist), keinen Nischenmarkt für die Rolle der privaten Unternehmen gibt. Ich denke, dass es einen Markt gibt, und dass dieser in Bezug auf seine massstäblichen Anpassungsmöglichkeiten gewinnbringend sein kann; jedoch bleibt noch die Frage, wer diese

2 Heeks Richard, Information and Communication Technologies, Poverty and Development, Development Informatics Working Paper Series, $\mathrm{n}^{\mathrm{o}}$ 5, Manchester, Institute for Development Policy and Management (University of Manchester), 1999, <http://idpm.man.ac.uk/publications/wp/di/di_wp05. pdf $>$, S. 17. 
Gebiete identifizieren wird. Hier setzt die Rolle der Intermediäre ein, die mit diesen besonderen Gebieten am besten vertraut sind. Diese Intermediäre könnten Nichtregierungsorganisationen (NRO), Gemeinschaftsgruppen, staatliche Organisationen usw. sein. Das Dilemma ist jedoch, dass diese Akteure durch zu viel privatwirtschaftliche Intervention zwangsläufig im Mechanismus des Marktes verfangen werden. Wenn die ärmeren Gebiete und Gemeinschaften nur den Marktkräften überlassen werden, dann werden sie sich niemals durch IKT-Lösungen emanzipieren können, da die privaten Unternehmen niemals den Besitz oder die Beteiligung an Lösungen zulassen werden, die für die ärmeren Gemeinschaften gemacht sind. Ich glaube, dass in diesem Fall die Lösung darin besteht, soziales Unternehmertum $\mathrm{zu}$ entwickeln. Wenn eine Gemeinschaft befähigt werden kann, Lösungen zur Verbesserung ihrer Lebensbedingungen umzusetzen - wobei diese Lösungen verhandlungsfähige Dienstleistungsgebühren mit einschliessen können so kann sie einen langen erfolgreichen Weg gehen. Viele IKT-Basisinitiativen, die derzeit in der Durchführung begriffen sind und von der betreffenden Gemeinschaft weiterhin unterstützt werden, werden schliesslich den Beweis hierfür erbringen.
Ein umfassender Zugang kann nicht durch private Investitionen gesichert werden. Geht es beim Zugang um die Infrastruktur, so sollte in erster Linie die Regierung mit der Unterstützung anderer Intermediäre einschreiten. Zuweilen gibt es einige innovative Ansätze, jedoch kann nur die Regierung eine Durchführung in grossem Massstab erreichen. Geht es beim Zugang um den Inhalt und die Emanzipation der Gemeinschaft, so gibt es viele Akteure, die ebenfalls einbezogen werden können. Die hauptsächlichen Massnahmen müssen von den $\mathrm{NRO}$, den Regierungen und anderen Gruppen getroffen werden, die Privatwirtschaft kann in der letzten Phase des Prozesses eingeschaltet werden. Ein wichtiger Faktor wäre, zwischen allen Akteuren ein Gleichgewicht aufrechtzuerhalten, so dass die neuen Technologien (einschliesslich jener mit Markennamen), die dazu bestimmt sind, den Bedürfnisse der ärmeren Gemeinschaften nachzukommen, diese Gruppen erreichen. Dabei könnten die Gemeinschaften sogar in einer bestimmten Phase für die Aufrechterhaltung dieser Dienstleistung bezahlen, die Teil ihrer sozialen und wirtschaftlichen Möglichkeiten ist. Ich glaube, dies könnte ein wichtiger Bereich für ein gemeinsames Vorgehen der internationalen Zusammenarbeit und des öffentlichen Sektors sein. 